\title{
ADVANCED DRILLING SYSTEMS
}

\author{
K. G. Pierce \\ Strategic Studies Center \\ Sandia National Laboratories
}

\author{
B. J. Livesay $m b \operatorname{lom}$ \\ Livesay Consültants \\ APR 081850
}

J. T. Finger

Geothermal Research Department

Sandia National Laboratories
This paper discusses the methods and results of a study of advanced drilling systems sponsored jointly by the Department of Energy Geothermal Division and the Natural Gas Technology Branch, Morgantown Energy Technology Center. Work performed at Sandia National Laboratories is supported by the U.S. Department of Energy under contract DE-AC04-94AL8500.

\section{Introduction}

Drilling is ubiquitous in oil, gas, geothermal, minerals, water well, and mining industries. Worldwide expenditures in oil and gas drilling approach $\$ 75$ billion per year. Lower cost wells could make it economically viable to exploit low yield and depleted oil and gas reservoirs. Drilling and well completion account for $25 \%$ to $50 \%$ of the cost of producing power from geothermal energy. Reduced drilling costs will reduce the cost of electricity produced from geothermal resources.

Attempts to improve or replace rotary drilling technology date back at least to the 1930's. Many novel and even exotic concepts were examined in the 1960's and 1970's and there has been some continuing effort through the 1980's. Much of this effort is documented in two books by Bill Maurer: Novel Drilling Techniques (1968) and Advanced Drilling Techniques (1980).

Undoubtedly, there are concepts for advanced drilling systems that have yet to be studied. However, the breadth and depth of previous efforts in this area almost guarantee that any new efforts will at least initially build on an idea or a variation of an idea that has already been investigated. Therefore, a review of previous efforts, coupled with a characterization of viable advanced drilling systems and the current state of technology as it applies to those systems, provide the basis for this study.

\section{A Systems Approach}

Nearly all studies of advanced drilling systems concentrate on methods of reducing rock. There is often little or no discussion of how these methods would fit into the full system necessary to drill, maintain, and complete a well. Unless the entire system is considered, much effort and money could be spent improving specific aspects of drilling technology only to discover that other facets of the problem prevent successful deployment of the system. Consequently, this study has not just investigated novel methods for reducing rock, but has examined all aspects of drilling systems necessary to drill and maintain a wellbore.

The following six functions must be performed by all drilling systems. The last element is not a necessary function in the sense that a well could be drilled without completion. However, completion is considered a basic function because (1) it is necessary for a well to be of any use and (2) it is a significant part of the cost of a well.

\begin{tabular}{l}
\hline \multicolumn{1}{c}{ Basic Drilling Functions } \\
- Energy transmission to the system-rock \\
interface \\
- Rock reduction \\
- Debris removal \\
- Borehole maintenance while drilling \\
- Completion \\
\hline \hline
\end{tabular}

In addition to the above drilling functions, any system must also operate under at least the 
following technical requirements and institutional constraints.

\section{System Constraints}

- Environmental impact

- Operational safety

- Government regulations

- Directional drilling and control

- Sensing and communication

While we have generally classified drilling systems according to cutting mechanism, we have analyzed the systems according to how they perform the basic drilling functions under the constraints listed above.

The following list gives the concepts and systems, sorted by cutting mechanism, that have been studied. This list was developed to cover the range from current technology, through ongoing efforts in drilling research, to highly speculative concepts. Included are cutting mechanisms that induce stress mechanically, hydraulically, and thermally.

\section{Systems and Concepts}

- Conventional rotary technology

- Coiled tubing drill rig

- Rotary-assist:

$\rightarrow$ Jet-assist

$\rightarrow$ Projectile-assist

$\rightarrow$ Thermal-assist (microwave)

- Mud hammer

- Thermal spallation

- Jet drilling

- Spark drill

- Explosive drill

- Rock melters:

$\rightarrow \quad$ Electric heater

$\rightarrow \quad$ Laser thermal

$\rightarrow$ E-beam

$\rightarrow$ Plasma arc

- Pulsed-laser water-jet

Most, if not all, of the concepts listed above will be familiar to anyone who has followed the efforts in the development of novel drilling systems. The only concept less than twenty years old is the pulsed-laser water-jet which has been proposed and investigated by PowerPulse Systems of Lakewood, CO (ref 3).

Many of the concepts are currently being studied or developed. At least three companies; FlowDril in Kent, WA (ref 4), Maurer Engineering in Houston, TX (ref 5), and TeleJet Technologies in Dallas, TX (refs 6 and 7); are actively pursuing jet-assisted drilling. Tround International of Washington, DC, has an operational projectileassisted drilling system (refs 8 and 9). Tetra Corporation (ref 10) in Albuquerque, NM, is studying the use of spark discharge for reducing rock.

Novatek in Provo, UT, has an operational mud hammer (ref 11). Researchers at MIT Energy $\mathrm{Lab}$ and at Los Alamos National Laboratory (LANL) have continued to study thermal spallation (ref 12); and Worldrill has applied for a European patent on a system to reduce the number of necessary conduits (ref 13). LANL also has a program studying the use of a rock melting system for environmental drilling (ref 14).

\section{Methodology}

The initial phase of this study was general information and data gathering. During this time, we established an initial set of concepts and identified individuals and organizations involved with each concept.

We developed descriptions based on the six drilling functions and completed system layouts. Based on the layouts, we defined equipment and material requirements and began to identify the strengths and weaknesses of each system.

During this same time, we initiated a series of technical discussions with individuals both currently and previously involved in advanced drilling research. We engaged in two primary types of discussions:

- There were general discussions where we presented the project organization, direction, and goals and invited comments and criticisms; and

- There were discussions of technical details concerning current and past research efforts on specific concepts and systems. 
Throughout this period we collected cost and performance data not only on advanced drilling systems, but also on conventional rotary technology. We developed a number of models and analysis routines relating cost to performance requirements and used these models to compare each system to current technology.

\section{Operating Costs}

There are many ways to assess the viability of advanced drilling concepts. Instead of concentrating only on technical feasibility we also estimated the capital and operating costs of advanced systems. Due to excess equipment and low demand, rig rates today are artificially low. Thus, it was necessary to estimate the costs of a conventional rotary drilling system built from all new equipment and materials as a basis for equitable comparisons to the expected costs of advanced systems. Using these cost estimates, we calculated the performance required for the advanced systems to be economically competitive with conventional rotary drilling.

We estimate that it would cost about ninemillion dollars to build and field an 18,000-foot conventional rig from all new equipment and materials. The rental rate for this rig alone would be about $\$ 12,900$ per day. There would be an additional $\$ 6,200$ per day in operator-incurred drill-site charges for a total daily rate of $\$ 19,100$. Current daily rates for an 18,000-foot rig are about $\$ 13,200$ ( $\$ 7,000$ rig rate plus $\$ 6,200$ additional drilling costs to the operator). We estimated performance requirements for advanced systems competing both with existing rigs and with newlybuilt rigs which are more expensive but represent the future market.

\section{Performance Requirements}

Performance assessment for most advanced concepts is difficult. The technical maturity varies dramatically from concept to concept. Data for some systems include field tests, while other systems have not progressed beyond bench tests, and still others have yet to be tested as a system in any format. It is neither easy nor accurate to extrapolate expected performance characteristics from such data.

Instead of estimating performance capabilities, we estimated performance requirements.
These requirements are based on the necessary penetration rate and life such that the advanced technology will cost no more than conventional rotary technology over a specific drilling interval.

The drilling interval chosen is a $121 / 4$-inch hole from 4,000 feet to 8,000 feet, completed with $95 / 8$-inch casing. We considered three general rock types defined as soft (IADC Series 51x and 52x), medium (IADC Series 53x through 61x), and hard (IADC Series 62x through 74x). The penetration rates and bit lives assumed for current technology in each of these formations are given in the following table.

\begin{tabular}{lcc}
\hline \hline \multicolumn{3}{c}{ Assumed Rotary Bit Performance } \\
\hline & ROP & Life \\
Soft & $40 \mathrm{fph}$ & $90 \mathrm{hr}$ \\
Medium & $15 \mathrm{fph}$ & $90 \mathrm{hr}$ \\
Hard & $7 \mathrm{fph}$ & $90 \mathrm{hr}$ \\
\hline \hline
\end{tabular}

In estimating interval costs, we included time and materials associated with drilling, hole conditioning, logging, casing, cement, testing, and well control. Under the requirement that the advanced technology cost no more than current rotary drilling, the result is minimum rate-ofpenetration as a function of equipment life.

Most of the concepts we considered could be introduced to drilling either as rental tools or as capital equipment. To evaluate the concepts for either contingency required a method of estimating rental rates. We used a cash flow analysis that considered interest rate, capital investment, repair costs, mean-time-between-repair, expected life, idle time (time not on the meter), operational overhead, and profit margin. The rental rate was estimated under the requirement that income cover costs over the life of the tool. Profit margin was included as a percentage of costs and stand-by charges were estimated to cover capital expenses only.

Based on the methodology and the assumptions discussed in the previous paragraphs, the table at the end of this paper summarizes the estimated performance requirements.

\section{Conventional Rotary Performance}

We developed performance requirements for various systems and concepts under the constraint 
that drilling with these systems cost no more than drilling with conventional rotary technology. It is also informative to examine the performance of conventional drilling technology.

The breakout of the costs incurred in completing the defined interval with conventional technology is illustrated in the following figure.

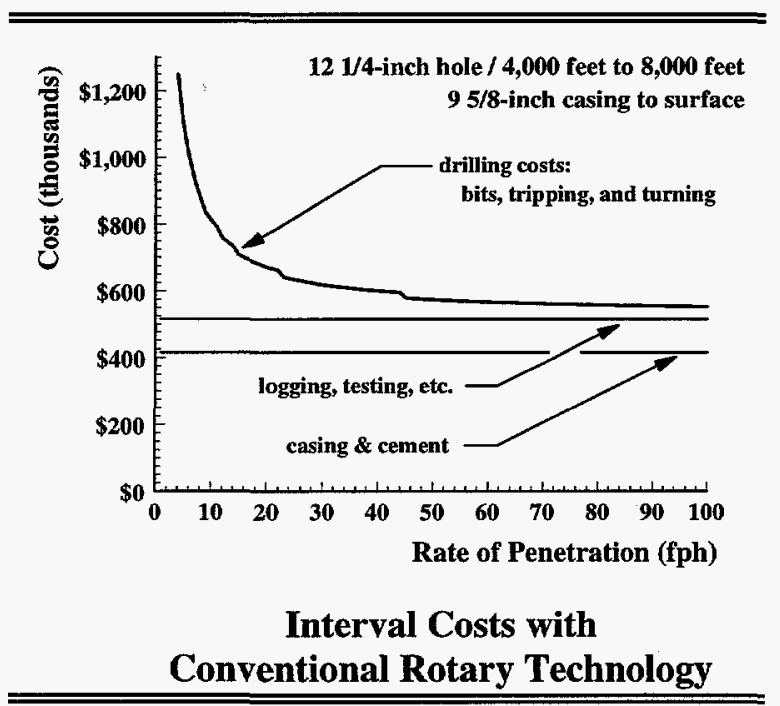

The end-of-interval costs (i.e. casing, cement, logging, testing, etc.) do not vary with penetration rate. However, the costs of drilling (bits, tripping, and turning on bottom) vary significantly with penetration rate. Most of the systems and concepts we investigated would affect the costs of drilling.

\section{Costs and Possible Savings}

The performance requirements were developed under the constraint that the advanced technology cost no more than current technology in completing the defined interval. Another approach would be to estimate savings given a particular improvement in penetration rate.

In most cases, merely matching current performance would be insufficient for a system to achieve commercial success. A system would need to surpass current performance in order to earn acceptance in the drilling industry. Based on the same 4,000-foot drilling interval used previously, the figure below shows savings in dollars that could be realized if the penetration rate is doubled or quintupled while all other factors are held constant.

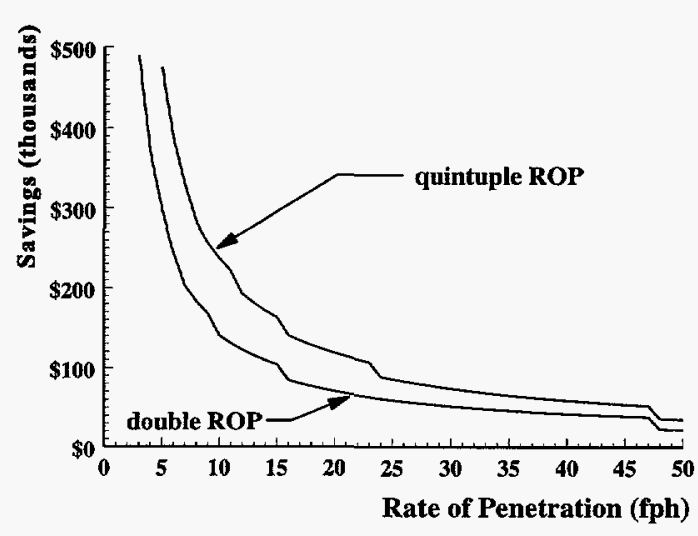

Possible Savings Through Improved Performance

As an example of how to interpret this figure, consider the possible savings at an ROP of $20 \mathrm{fph}$. This figure indicates that doubling the ROP would result in savings of about $\$ 70,000$, while quintupling the ROP would yield savings on the order of $\$ 120,000$.

The possible savings increases significantly as penetration rates decrease below fifteen to twenty feet-per-hour. This region is a particularly attractive target for systems whose primary advantage is to increase the rate of penetration.

Based on the previous figures and discussion, the greatest opportunity for reducing costs through improved rock cutting techniques is in hard-rock drilling. That has been the experience of people who have attempted to market new techniques for cutting rock.

\section{Common Problems}

We took a systems approach to avoid overlooking some facet of the problem that would prevent successful deployment of a system; however, there has been another consequence of the systems approach: we identified a number of common problems that run across multiple systems. The concept of common problems is significant in that the solution to one of these could advance the viability of all the systems cross-cut by that problem. These common problems include the following:

- Multi-channel conduit,

- Electric conductor downhole, 
- Maintenance of the borehole gage,

- Maintenance of stand-off distance,

- Well control and wellbore stability in the absence of liquids,

- $\quad$ Reduced effectiveness with depth, and

- The size of the surface system.

\section{Multi-Channel Conduit}

A number of the systems under consideration require multiple conduits for the transmission of different fluids and/or electrical energy. Multiconduit pipe can be manufactured. FlowDril used dual-wall pipe for their system and TeleJet Technologies has designed multi-conduit pipe for the MultiCon $^{\mathrm{TM}}$ system. Concentric drill pipe is available commercially in the U.S. When compared to standard drill pipe, it is generally heavy, expensive, and difficult to handle.

\section{Electrical Transmission}

A number of systems would benefit from cheap and reliable methods to transmit electricity to the drill head. This is especially true for highenergy systems. Even rotary technology would benefit from such a development. A power cable would allow the use of electric motors, actuators, and control systems. The development of fast, reliable telemetry would allow not only the use of current downhole sensors such as pressure, temperature, and formation evaluation tools; but also the development and use of systems to evaluate the condition of the bit, to detect kicks almost instantaneously, and to provide data for real-time analyses of downhole conditions.

\section{Borehole Gage and Trajectory Control}

Maintenance of borehole gage and trajectory is a concern for nearly all of the system concepts that are not rotary hybrids. For a given set of conditions, the diameter of the hole created with high-pressure jets and thermal systems will depend largely on the advance rate of the drilling head. There is a minimum hole diameter needed for running casing. Above this minimum, though, excessive variation can cause problems when cementing the casing.

\section{Control of Stand-Off Distance}

The efficiency of most systems that do not maintain direct contact with the rock is dependent on stand-off. A simple solution is a mechanical probe, but some systems may require a more elaborate mechanism. It is not clear that a universal stand-off control system, cross-cutting several systems can be developed.

\section{Well Control and Wellbore Stability}

A number of the concepts investigated cannot operate under a full column of liquid. While cuttings can be removed with air, the absence of drilling mud greatly inhibits the ability to control formation fluids. Also, the contributions of drilling muds to wellbore stability through static pressure and chemical additives are lost. The applicability of any system that cannot operate in the presence of drilling mud is diminished.

In connection with the principle that most formations drill faster with less wellbore pressure, a quick way to increase penetration rate with rotary technology is to lighten the drilling mud even to the point of drilling underbalanced. Improvement of methods to control formation fluids and maintain borehole stability while drilling underbalanced could significantly increase penetration rate and reduce drilling time.

\section{Reduced Effectiveness with Depth}

As with conventional drilling, several of the advanced concepts have demonstrated reduced cutting effectiveness with depth. Although this effect may not be universal, it is common enough to suggest that there is still a need for better understanding of depth and fluid pressure effects on rock properties as they apply to drilling.

More importantly, these experiences imply that a first step in the development of any new drilling system should be to test the performance of the concept at depth. Existing facilities can independently simulate pore pressure, rock stress, and borehole fluid pressure at depth. Unconventional rock-cutting concepts can be tested at one of these facilities prior to the expenditure of significant resources on system development.

\section{Size of the Surface System}

For a land-based rig, the capital investment in the surface system is about nine-million dollars. The size, cost, and complexity of the drill rig's surface system is little affected by the way we cut rock. 
The sizes and specifications for the mast, substructure, and drawworks are determined by the need to handle casing. The requirements of the mud pumps, pits, and mud-cleaning equipment are determined by the size of the cuttings and the rate at which they are produced. About the only equipment that depends on how we cut rock is the bottom hole assembly. It is doubtful that any novel rock cutting mechanism will cost less than drill collars, stabilizers, and bits. Overall, it is unlikely that significant savings in materials and equipment can be achieved by simply changing the way we cut the rock.

Similar conclusions are reached when daily operational costs are considered. The numbers and skills of the crew are determined by the surface equipment. Rig insurance is determined by capital investment; liability insurance and workman's compensation costs are proportional to payroll.

Reduction of drilling costs can occur only by changing the nature of the surface system or increasing the rate of penetration. Neither capital investment nor daily operational costs are significantly affected by the way we cut rock. Any increase in capital or operating costs must be offset by a commensurate increase in penetration rate. Unconventional rock-cutting mechanisms can reduce costs only if they can increase the rate of penetration.

\section{Summary}

The authors wish to thank the sponsors for their support throughout this study. This paper has presented an overview of the methods and a summary of results of a study of advanced drilling systems. Much greater detail will be included in the final report. We hope that these efforts will be of use to project managers and policy makers in making decisions concerning the expenditure of resources for the development of drilling systems.

\section{References}

1. William C. Maurer, Novel Drilling Techniques, Pergamon Press, Library of Congress No. 68-17738, 1968

2. William C. Maurer, Advanced Drilling Techniques, Petroleum Publishing Company, Tulsa, OK, ISBN 0-87814-117-0, 1980
3. John G. Sellar, Rock Excavation Using a Pulsing Laser-Water Jet, report prepared for the EPRI Center for Materials Production, Pittsburgh, PA, CMP report number 93-7, November 1993

4. Scott D. Veenhuizen, FlowDril Corporation, DOE/GRI Development and Testing of a Downhole Pump for Jet-Assist Drilling, Proceedings of the Natural Gas Contractors RD\&D Review Meeting, April 1995

5. John H. Cohen, Gefei Liu, and Curtis E. Leitko, Maurer Engineering, John Aslakson, Gas Research Institute, Steve Kennedy and Lawrence Lee, REDA Pump Company, HighPressure Pump for Jet-Assisted Drilling, ASME, PG-Vol. 65 Drilling Technology, Book No. H00920-1995

6. Frank J. Schuh, MultiCon ${ }^{\mathrm{TM}}$ Drilling System Potential, Drilling Technology, Inc., Plano, TX, February 1994

7. Frank J. Schuh, MultiCon ${ }^{\mathrm{TM}}$ Drilling System Economics, Drilling Technology, Inc., Plano, TX, January 1995

8. Robert J. Regan, Mining Cannon is a Real Rockbuster, Iron Age, June 24, 1983

9. Allen Howland, Machine Gun Bit Speeds Air Drilling, Drilling Technology, August 1984

10. William M. Moeny and James G. Small, Focused Shock Spark Discharge Using Multiple Electrodes, U.S. Patent \#4,741,405, May 1988

11. David S. Pixton, Yu Xiangguang, Vance I. Fryer, Steven J. Payne, David R. Hall, A New Generation Mud Driven Rotary Percussion Tool: Summary of Current Research and Development Efforts, Second Ed., Novatek, Provo, UT, February 1990

12. Jefferson W. Tester, Robert M. Potter, Carl R. Peterson, Howard J. Herzog, MIT, John North, Worldrill, and John E. Mock, Geothermal Division U.S. DOE, Advanced Drilling and Its Impact on Heat Mining

13. Worldrill Corporation, Improvements in or Relating to Drilling with Centrifuge/Vortex Combustion Jet Spallation, British and Foreign Patent Application, continuation of patent application PCT/GB94/00515 
14. Sue J. Goff, Gilles Y. Bussod, Kenneth Wohletz, Aaron Dick, and John C. Rowley, Rock Melting: A Specialty Drilling System for Improved Hole Stability in Geothermal Wells, Los Alamos National Laboratory, Los Alamos, $\mathrm{NM}$

\begin{tabular}{|c|c|c|c|}
\hline \multicolumn{4}{|c|}{ Summary of ROP Requirements ${ }^{1}$} \\
\hline & Soft & Medium & Hard \\
\hline $\begin{array}{l}\text { Fully integrated coiled-tubing rig } \\
\text { Jet-assist }\end{array}$ & $1.5-3.5$ & $1.4-2.3$ & $1.4-2.1$ \\
\hline Surface pressure generation & $>3.4$ & $2.2-5.0$ & $1.9-3.4$ \\
\hline Positive-displacement DHP & $1.7-2.0$ & $1.6-1.9$ & $1.6-1.9$ \\
\hline Centrifugal DHP & $1.6-1.8$ & $1.5-1.7$ & $1.5-1.7$ \\
\hline Projectile-assist & $2.2-3.2$ & $2.0-2.5$ & $1.9-2.3$ \\
\hline Microwave-assist & $?$ & $?$ & $?$ \\
\hline Mud hammer & $1.4-1.6$ & $1.3-1.5$ & $1.3-1.5$ \\
\hline Thermal spallation & & & \\
\hline Downhole separation & $1.1-1.4$ & $1.1-1.3$ & $1.1-1.2$ \\
\hline Spark drill & $?$ & $?$ & $?$ \\
\hline Explosive drill & $?$ & $?$ & $?$ \\
\hline Pulse laser-water jet $(3,500-\mathrm{hr}$ life $)$ & $\sim 2.5$ & $\sim 1.7$ & $\sim 1.5$ \\
\hline Rock melters & $?$ & $?$ & $?$ \\
\hline
\end{tabular}

